\title{
Endovascular treatment of carotid artery restenosis
}

Viroj Wiwanitkit ${ }^{1}$

Editor, I have read the recent report on "Endovascular treatment of carotid artery restenosis" with a great interest $^{1}$. Lujan et al. concluded that "Endovascular treatment of carotid artery restenosis seems to be a feasible and safe approach in the short term ${ }^{1}$." This work is limited by its nature of retrospective study with few subjects. The short-term success can be derived, but this might be due to the experience of the practitioner, good patient selection, or good instrument and it might not imply the efficacy and safety of the method. Indeed, in some large studies, a complication, such as embolism, can be seen and this should be the major concern of selecting this technique ${ }^{2}$.

\section{References}

1. Lujan RAC, Luca LA, Garcio AF, Carvalho GML, Lobato AC. Endovascular treatment of carotid artery restenosis: short term results. J Vasc Bras. 2011;10(1):3-8.

2. Matsumaru Y, Hayakawa M. Endovascular treatment for carotid artery stenosis. Brain Nerve. 2010;62(12):1291-300. 